\title{
ASTRID: Accurate Species TRees from Internode Distances
}

\author{
Pranjal Vachaspati, Tandy Warnow* \\ From 13th Annual Research in Computational Molecular Biology (RECOMB) Satellite Workshop on \\ Comparative Genomics \\ Frankfurt, Germany. 4-7 October 2015
}

\begin{abstract}
Background: Incomplete lineage sorting (ILS), modelled by the multi-species coalescent (MSC), is known to create discordance between gene trees and species trees, and lead to inaccurate species tree estimations unless appropriate methods are used to estimate the species tree. While many statistically consistent methods have been developed to estimate the species tree in the presence of ILS, only ASTRAL-2 and NJst have been shown to have good accuracy on large datasets. Yet, NJst is generally slower and less accurate than ASTRAL-2, and cannot run on some datasets.

Results: We have redesigned NJst to enable it to run on all datasets, and we have expanded its design space so that it can be used with different distance-based tree estimation methods. The resultant method, ASTRID, is statistically consistent under the MSC model, and has accuracy that is competitive with ASTRAL-2. Furthermore, ASTRID is much faster than ASTRAL-2, completing in minutes on some datasets for which ASTRAL-2 used hours.

Conclusions: ASTRID is a new coalescent-based method for species tree estimation that is competitive with the best current method in terms of accuracy, while being much faster. ASTRID is available in open source form on github.
\end{abstract}

\section{Background}

Species tree estimation in the presence of gene tree incongruence is a major challenge for many biological analyses. Gene tree incongruence can result from a variety of processes, notably incomplete lineage sorting (ILS) [1], which is modelled by the multispecies coalescent (MSC) [2]. Concatenated maximum likelihood analyses is generally the most common method for species tree estimation from multiple loci, but can be statistically inconsistent, and even positively misleading, in some cases [3], thus converging to an incorrect tree with increasing amounts of sequence data.

In recent years, a number of species tree estimation methods have been developed that are statistically consistent under the MSC, and so will converge in probability to the true species trees as the amount of data increases; see [4-6]. Methods that are statistically consistent under the MSC include ASTRAL [7], ASTRAL-2 [8], "BEAST [9],

\footnotetext{
* Correspondence: warnow@illinois.edu

Department of Computer Science, University of Illinois at UrbanaChampaign, 201 N. Goodwin Avenue, Urbana, IL, 61801 USA
}

BEST [10], the population tree from BUCKy [11], METAL [12], MP-EST [13], NJst [14], SNAPP [15], STEAC [16], STEM [17], and SVDquartets [18]. While little is yet known about some of these methods (either because they have not yet been adequately studied or because they are not yet implemented), only a few of them (MP-EST, NJst, and ASTRAL-2) have been shown to be able to analyze very large datasets (especially those with large numbers of taxa) with high accuracy. MP-EST has been used more than either NJst or ASTRAL-2, but NJst is more accurate than MP-EST, and ASTRAL-2 is more accurate than both [8]. Furthermore, the currently available implementation of NJst is slower than ASTRAL-2, and cannot run on some datasets $[8,14]$.

In this paper, we present ASTRID, a new ILS-aware distance-based method for species tree estimation. Our approach is based on NJst, but is substantially faster, and, unlike NJst, functions even when each gene tree contains only a small portion of the data. The input to NJst is a set of unrooted gene trees. In the first step, an $n \times n$ matrix $D$ $[x, y]$ is computed, where $D[x, y]$ is the average distance 
(in terms of number of edges) between $x$ and $y$ among all the gene trees. In the second step, neighbor joining [19], a very popular distance-based method of phylogeny estimation, is used to produce the species tree.

ASTRID improves on NJst by enabling other distancebased methods to be used in the second step. In particular, although NJ cannot be run on datasets with missing entries, other distance-based methods can, and ASTRID enables the use of these other methods. We also explore the use of more accurate distance-based methods. Thus, ASTRID is a very simple modification to NJst. As we will show, ASTRID is much faster than NJst.

The comparison between ASTRID and ASTRAL-2 and MP-EST, two established coalescent-based summary methods, is also interesting. ASTRID completed in minutes on some datasets where the other methods took hours, and was fast enough to analyze datasets with 1000 species and 1000 genes on a single processor within an hour (ASTRAL-2 and MP-EST take much more time on datasets of this size). Furthermore, ASTRID clearly dominates MP-EST in terms of accuracy, and is competitive with ASTRAL-2 (more accurate in some cases, and less accurate in others). Finally, ASTRID has desirable theoretical properties: it runs in polynomial time, and it remains statistically consistent under the MSC model without assuming the molecular clock, nor requiring rooted gene trees as input.

\section{Methods}

\section{ASTRID}

The input to ASTRID is a set of unrooted gene trees $T_{1}, \ldots$, $T_{k}$. We let $S=L\left(T_{i}\right)$ denote the leafset of $T_{i}$, and $S=\cup_{i} L\left(T_{i}\right)$. Let $|S|=n$.

Step 1: Construct $n \times n$ matrix $\bar{M}$ :

1 For all $i=1,2, \ldots, k$, compute $n \times n$ matrix $M_{i}$, as follows. For pairs $p, q$ of species where both are in $S_{i}$, set $M_{i}(p, q)$ to be the number of edges in the path between $p$ and $q$ in $T_{i}$. For all other pairs $p, q$ (i.e., where one or both are not in $\left.S_{i}\right)$, set $M_{i}(p, q)=0$. Thus, the only non-zero entries in $M_{i}$ are for pairs of species in $T_{i}$.

2 For all $\{p, q\} \subset S$, let $n(p, q)$ be the number of trees $T_{i}$ that contain both $p$ and $q$.

3 Define $n \times n$ matrix $\bar{M}$ by setting $\bar{M}(p, q)=\frac{\sum_{i} M_{i}(p, q)}{n(p, q)}$ if $n(p, q)>0$, and $\bar{M}[p, q]=-1$

(to denote a missing value) otherwise.

Step 2: Compute tree on $\bar{M}$ using a selected distancebased method

\section{Datasets}

We tested species tree estimation methods on simulated datasets from previous publications, and also evaluated
ASTRID on the mammalian biological dataset of 37 species, originally studied in [20]. Here we briefly describe the simulation procedures used to generate these datasets, and provide empirical statistics for the datasets in Table 1. See the original publications for details about the simulation protocols, and our supplementary online materials for links to the data.

All datasets included both true and estimated gene trees, obtained by using maximum likelihood methods on the true sequence alignments, as well as species trees estimated on these gene trees obtained in the prior publications. Each gene tree had at most one copy of each species. We computed ASTRID species trees for these datasets, using various techniques for Step 2 (how to compute the species tree given the distance matrix).

We estimated the amount of ILS in the data by quantifying the average gene tree discord in the data, using the average Robinson-Foulds (RF) [21] distance between true gene trees and the model species tree, expressed as a percentage (written AD for "average distance"). We also explored some simulated datasets where the DNA sequence evolution was under the strict molecular clock. Model conditions with AD at most $25 \%$ can be considered low ILS, conditions with AD between $26 \%$ and $39 \%$ can be considered moderate ILS, conditions with AD between $40 \%$ and $59 \%$ can be considered high ILS, and conditions with AD of at least $60 \%$ can be considered very high ILS. In Table 1, we indicate these ILS levels for the different model conditions we study both with the AD value, but also the general level ( $\mathrm{L}$ for low, $\mathrm{M}$ for moderate, $\mathrm{H}$ for high, and $\mathrm{VH}$ for very high).

\section{Mammalian and avian simulated datasets}

These datasets were created in [22] to evaluate method performance under model conditions similar to real data. Species trees were generated with MP-EST for the avian phylogenomics dataset with 48 species and 14,446 loci [24], and for a mammalian dataset with 37 species and 447 loci [25]. These species trees were used as basic model trees, with branch lengths in coalescent units. In addition, two other model species trees were created for each dataset by scaling the species tree branch lengths up (to reduce ILS) or down (to increase ILS). The ILS levels of the resultant model species trees were very heterogeneous, ranging from $\mathrm{AD}=21 \%$ (low) to $50 \%$ (high) for the mammalian simulation, and from $\mathrm{AD}=29 \%$ (moderate) to $60 \%$ (very high) for the avian simulation.

Both datasets had sequences of length 500 for all three model conditions. For the default ("1X") branch length condition, the avian dataset also had sequences of length $250,500,100$, and 1500, and the mammalian dataset had sequences of length 250, 500 and 1000. Sequence evolution on these datasets deviated from the strict molecular clock.

\section{0-taxon simulated datasets}

These data were presented in [23], and explored two ILS levels $(\mathrm{AD}=48 \%$ (high) and $\mathrm{AD}=89 \%$ (very high)). 
Table 1 Empirical statistics of simulated datasets used in this study

\begin{tabular}{|c|c|c|c|c|c|}
\hline Dataset & \# genes & \# taxa & ILS level (AD\%) & \# sites & ref. \\
\hline Avian very high ILS $(0.5 X)$ & 1000 & 48 & $60(\mathrm{VH})$ & 500 & [22] \\
\hline Avian high ILS (1X) & 1000 & 48 & $47(\mathrm{H})$ & $250-1500$ & [22] \\
\hline Avian moderate $(2 X)$ & 1000 & 48 & $29(\mathrm{M})$ & 500 & [22] \\
\hline Mammalian high ILS (0.5X) & 200 & 37 & $50(\mathrm{H})$ & $250-1000$ & [22] \\
\hline Mammalian moderate ILS (1X) & 200 & 37 & $29(\mathrm{M})$ & $250-1000$ & [22] \\
\hline Mammalian low ILS (2X) & 200 & 37 & $21(\mathrm{~L})$ & 250-1000 & [22] \\
\hline 10-taxon very high ILS & 200 & 10 & $89(\mathrm{VH})$ & 100 & [23] \\
\hline 10-taxon high ILS & 200 & 10 & $48(\mathrm{H})$ & 100 & [23] \\
\hline 15-taxon clocklike & 1000 & 15 & $82(\mathrm{VH})$ & $100-1000$ & [23] \\
\hline ASTRAL-2 500K-1e6 (MC1) & 1000 & 200 & $69(\mathrm{VH})$ & $300-1500$ & {$[8]$} \\
\hline ASTRAL-2 2M-1e6 (MC2) & 1000 & 200 & $33(\mathrm{M})$ & $300-1500$ & {$[8]$} \\
\hline ASTRAL-2 10M-1e6 (MC3) & 1000 & 200 & $21(\mathrm{~L})$ & $300-1500$ & {$[8]$} \\
\hline ASTRAL-2 500K-1e7 (MC4) & 1000 & 200 & $68(\mathrm{VH})$ & $300-1500$ & {$[8]$} \\
\hline ASTRAL-2 2M-1e7 (MC5) & 1000 & 200 & $34(\mathrm{M})$ & $300-1500$ & {$[8]$} \\
\hline ASTRAL-2 10M-1e7 (MC6) & 1000 & 200 & $9(\mathrm{~L})$ & $300-1500$ & {$[8]$} \\
\hline ASTRAL-2 2M-1e6 (MC7) & 1000 & 10 & $17(\mathrm{~L})$ & $300-1500$ & {$[8]$} \\
\hline ASTRAL-2 2M-1e6 (MC8) & 1000 & 50 & $30(\mathrm{M})$ & $300-1500$ & [8] \\
\hline ASTRAL-2 2M-1e6 (MC9) & 1000 & 100 & $34(\mathrm{M})$ & $300-1500$ & {$[8]$} \\
\hline ASTRAL-2 2M-1e6 (MC10) & 1000 & 500 & $34(\mathrm{M})$ & $300-1500$ & {$[8]$} \\
\hline ASTRAL-2 2M-1e6 (MC11) & 1000 & 1000 & $35(\mathrm{M})$ & $300-1500$ & {$[8]$} \\
\hline
\end{tabular}

The ILS level is measured by the average Robinson-Foulds distance (AD) between the true gene trees and the species tree, expressed as a percentage; ILS levels are then classified as low $(\mathrm{L})$, moderate $(\mathrm{M})$, high $(\mathrm{H})$, or very high $(\mathrm{VH})$.

Sequence evolution deviated from the strict molecular clock.

\section{5-taxon clocklike simulated datasets}

These datasets evolved under a strict molecular clock, and were presented in [23]. The species tree was a caterpillar model tree (i.e., a path with leaves hanging off the path) with very short internal branches, and a long branch to the outgroup species. The ILS level in these data was very high $(\mathrm{AD}=82 \%)$.

\section{ASTRAL-2 simulated datasets}

These data were presented in [8], and provided a variety of model conditions with varying ILS levels, tree shapes, numbers of taxa, and sequence lengths per locus. SimPhy [26] was used to generate the species and gene trees, based on two parameters: the number of generations (given as the first number in the model) and the speciation rate (given as the second number). The number of generations simulated ranged between $500 \mathrm{~K}, 2 \mathrm{M}$, and $10 \mathrm{M}$, and the speciation rate varied between $1 \mathrm{e} 6$ and $1 \mathrm{e} 7$. Model conditions with fewer generations had more ILS. Model conditions with the 1 e 6 speciation rate had speciation events nearer the tips (leaves) of the trees, while model conditions with the $1 \mathrm{e} 7$ speciation rate had speciation events nearer the root. The ILS levels varied from very low $(A D=9 \%)$ to very high $(A D=69 \%)$. Sequences evolved down the gene trees under multiple GTRGAMMA models that deviated from the strict molecular clock. Maximum likelihood gene trees were computed using FastTree-2.

\section{Incomplete gene tree datasets}

To explore performance on incomplete gene trees, we modified the ASTRAL-2 dataset by randomly removing taxa from trees in the 50-taxon datasets. Up to 40 taxa were removed from the 50-taxon dataset, and up to 5 taxa were removed from the 10-taxon dataset. In each of these cases, maximum likelihood gene trees were estimated using FastTree-2 version 2.1.7 SSE3 [27], using the following command:

fasttree -nt -gtr-quiet - nopr -gamma - $n$ $1000<$ fastafile> > <genetreefile> where $<$ fastafile $>$ was the input file of aligned sequences and $<$ genetreefile $>$ was the output file.

\section{Distance-based tree estimation methods}

In order to explore the design space for ASTRID, we ran various distance-based methods for Step 2 (computing the tree from the distance matrix). For incomplete distance matrices (where some entries are -1 , indicating that the pair of taxa do not appear together in any gene tree), we explored the methods in PhyD [28]: $N J^{*}$, $B I O N J^{*}, M V R * U N J^{*}$. These algorithms are all variants on neighbor joining that work on incomplete distance matrices. We also explored FASTME [29], which is a heuristic for the minimum evolution problem. 


\section{ASTRAL-2}

To compute ASTRAL-2 species trees on the incomplete gene trees generated for the ASTRAL-2 datasets, we ran ASTRAL-2 version 4.7.8, using command line arguments

java-Xmx4000M -jar astral.4.7.8.jar -i <genetrees> - 0 <outputtree>

\section{Computing tree error}

All trees computed in this study were fully resolved. We report the RF tree error (the proportion of the branches in the model tree missing from the estimated tree), using scripts that are available in the supplementary online materials.

\section{Results}

Selection of distance-based tree estimation method for Step 2

First, we evaluated various distance-based tree estimation methods to determine which one would be most accurate for the tree computation phase of ASTRID. Results on datasets with all complete gene trees (no missing species in any gene) are shown in Figure 1 and results on datasets with incomplete gene trees are shown in Figure 2. Note that for datasets with entirely complete gene trees, FastME performed as well as or better than the other distance-based methods, but there were datasets with incomplete distance matrices in which FastME had very poor accuracy. Therefore, we selected FastME to analyze datasets where the distance matrix has no missing entries, since it had the best accuracy. For the datasets with incomplete distance matrices $\bar{M}$ (indicated by $\bar{M}[p, q]=-1$ for some $\mathrm{p}, \mathrm{q})$, we selected BioNJ", since it generally had among the most accurate results of these PhyD* methods.

\section{Comparison of ASTRID, ASTRAL, and MP-EST}

We begin with a comparison between ASTRID, ASTRAL-2, and MP-EST on the avian simulated datasets with high (1X) ILS, varying number of genes and sequence alignment lengths, but where all genes are complete; see Figure 3.

All methods improved with increasing numbers of genes or increasing sequence length; however, the methods differed substantially in terms of their accuracy. Across all conditions we explored, MP-EST had the highest error and ASTRID had the lowest error. ASTRAL-2 was in between, but was closer to ASTRID than to MP-EST. The gap between MP-EST and ASTRID was very large, and increased with the number of genes. For example, at 1000 genes and gene sequence alignments of length 500, MPEST had 19\% RF error while ASTRID had about 7\% RF error. The gap between ASTRID and ASTRAL-2 was substantial on the 200 -and 500 -gene cases, but very small on the 1000-gene case.

Thus, although MP-EST is statistically consistent under the MSC model and hence theoretically robust to ILS, it did not have particularly good accuracy on these data. Among all coalescent-based methods, MP-EST is probably the one that has been used the most in biological data analyses, but its performance here and in $[8,30]$ demonstrates that it is not competitive with the best methods on datasets with even moderate numbers of species. Therefore, we omit MP-EST from the rest of this study.

\section{Comparison of ASTRID and ASTRAL-2 on complete gene trees}

Comparison on avian datasets. Figure 4 shows the performance of ASTRAL-2 and ASTRID on avian simulated datasets under three ILS conditions (moderate, high, and very high). Both methods performed better

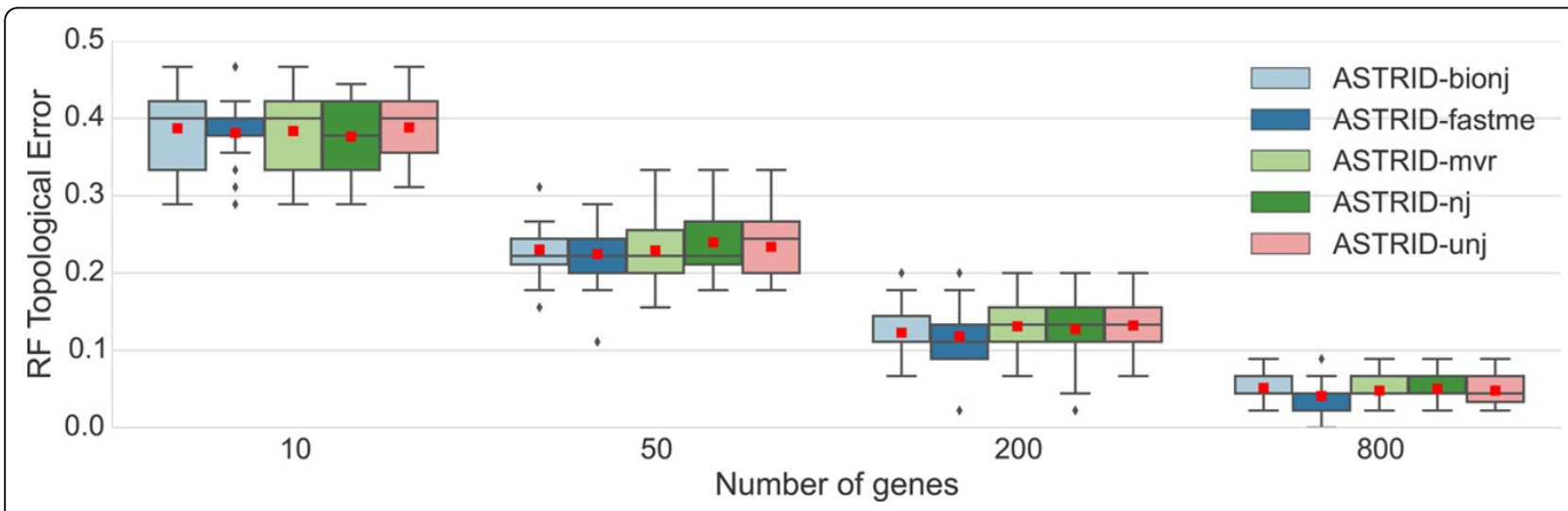

Figure 1 A comparison of ASTRID variants on the moderate ILS avian simulated datasets with $\mathbf{5 0 0}$ bp, using different distance-based methods for the tree estimation phase. We report RF topological error rates over 20 replicates. Red dots represent means, while lines represent medians and boxes represent quartiles. 

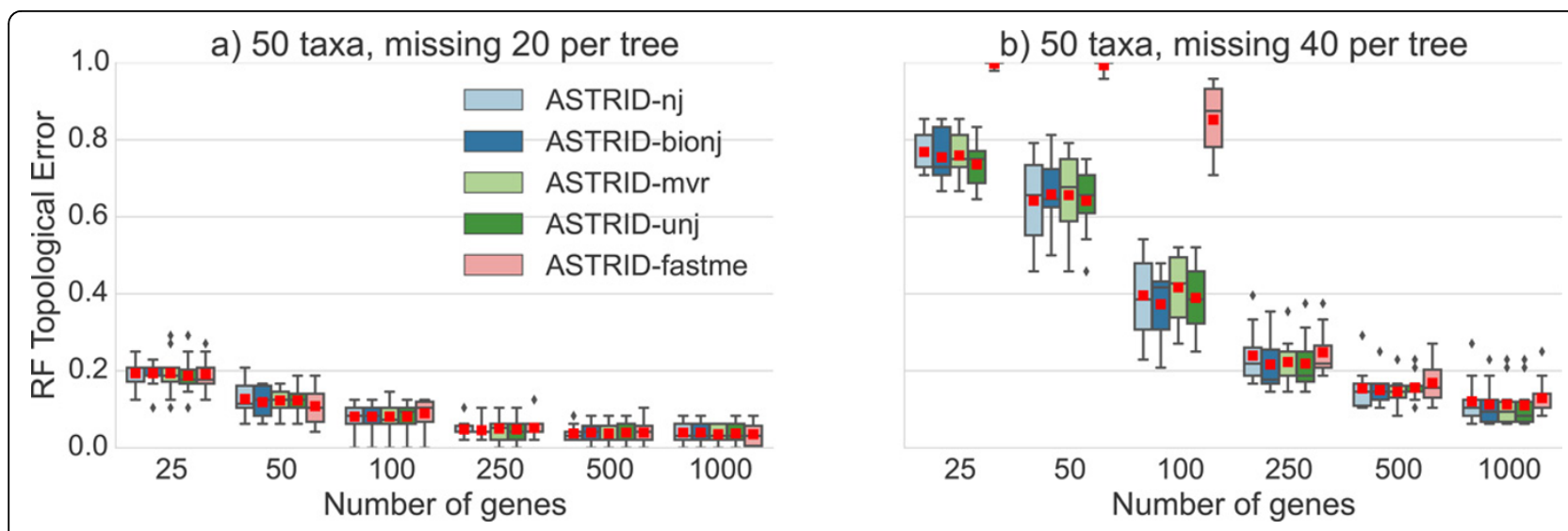

Figure 2 Comparison of ASTRID variants on 50-taxon ASTRAL-2 MC8 datasets with missing taxa. We show average RF error rates over 50 replicates for ASTRID variants, that differ in terms of the method used to compute the tree from the distance matrix. The datasets have taxa randomly removed from each gene and the sequence lengths truncated to $300 \mathrm{bp}$. Red dots represent means, while lines represent medians and boxes represent quartiles.
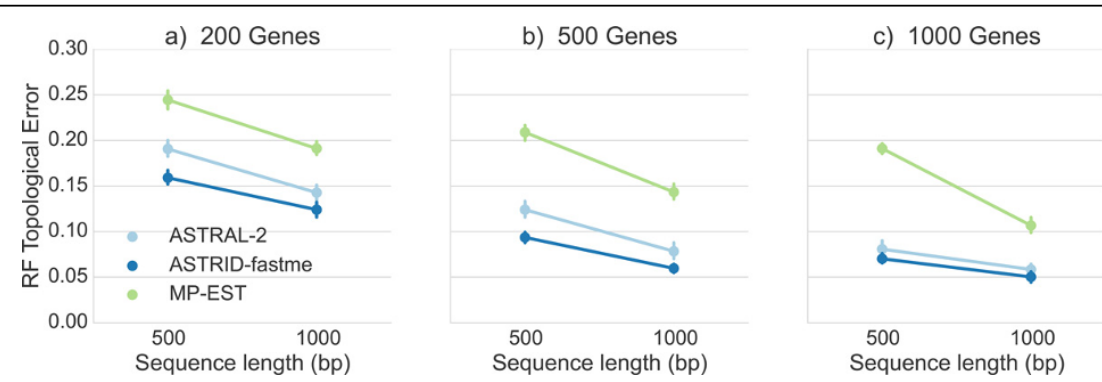

Figure 3 Comparison of ASTRID, ASTRAL-2, and MP-EST on the avian simulated data. The simulated data evolve under 1X (high ILS) species tree branch lengths, and with varying gene sequence lengths. We report mean RF rates with standard error bars over 20 replicates.
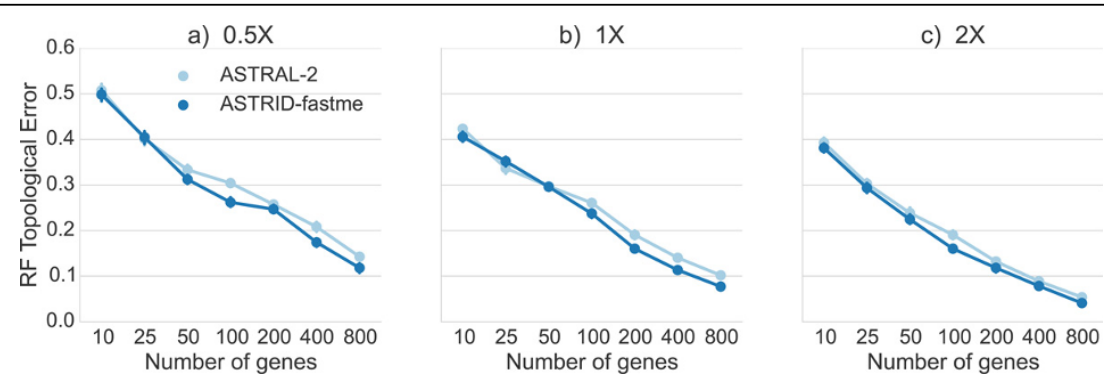

Figure 4 Comparison of ASTRID and ASTRAL-2 on avian simulated datasets. We show average RF error rates and standard error bars for 20 replicates. Gene sequence alignments have 500 sites and varying amount of ILS. Model conditions varied from very high ILS (0.5X) to moderate ILS (2X).

when provided with more genes, and both performed worse on higher levels of ILS. Overall, ASTRID tended to outperform ASTRAL-2, with the largest effect seen when many genes were available. With 800 genes available, the ASTRID species tree had a RF error rate that was 2.4 percentage points better than ASTRAL-2's under the very high and high ILS model conditions, and
1.2 percentage points better for the moderate ILS model condition. On the moderate ILS model condition, ASTRID had the greatest advantage over ASTRAL-2 for moderate numbers of genes. Above 200 genes, the error rate dropped below ten percent for both ASTRAL-2 and ASTRID, and ASTRID had an average advantage of only about one percentage point. 
It is well known that summary methods improve in accuracy as the number of sites per gene or the number of genes increase [31-34]. We explored the impact of varying the sequence length and number of genes on the avian datasets with high (1X) ILS, as well as on true gene trees. Figure 5 shows results on 10,100, and 1000 genes; results on other numbers of genes have the same trends (data provided in supplementary materials). As expected, both methods improved with increased sequence length, and had their best accuracy on true gene trees. Both methods also improved as the number of genes increased. ASTRID was always at least as accurate as ASTRAL-2, with the biggest improvement for shortest sequences (with $250 \mathrm{bp}$ ).

Comparison on mammalian datasets. A comparison of ASTRAL-2 and ASTRID on the mammalian datasets with different levels of ILS (high, moderate, and low) is given in Figure 6. ASTRAL-2 and ASTRID performed fairly similarly on the low (2X branch lengths) and moderate (1X branch lengths) ILS conditions. Under the high ILS level ( $0.5 \mathrm{X}$ branch lengths), ASTRAL-2 was fairly consistently more accurate than ASTRID, with the largest improvement on the 10-gene case.

Comparison on the ASTRAL-2 datasets. We explored performance on the ASTRAL-2 datasets with 200 taxa (model conditions MC1 to MC6, see Figure 7). These model trees varied in ILS level, with MC1 and MC4 having very high ILS, MC2 and MC5 having moderate ILS, and MC3 and MC6 having low ILS. Under MC2, MC3, and MC5, the two methods had essentially identical accuracy. However, under MC1, MC4, and MC6, ASTRAL-2 had an advantage over ASTRID. In MC1 and MC4, the improvement disappeared at 100 genes, but in MC6 ASTRAL-2 was still more accurate than ASTRID on 100 genes.

Comparison on the 15-taxon datasets. The 15-taxon datasets evolved on a caterpillar species tree under very high ILS ( $\mathrm{AD}=82 \%$ ), the highest ILS considered in this study. We explored performance under two sequence lengths (100 bp and $1000 \mathrm{bp}$ ) and varied the number of genes from 10 to 1000 . Results on the 15-taxon datasets (Figure 8) showed very close performance between ASTRID and ASTRAL-2. On the $100 \mathrm{bp}$ alignments and on $1000 \mathrm{bp}$ alignments with at least 100 genes, the two methods could not be distinguished. However, on 1000 bp alignments with at most 50 genes, ASTRAL-2 had an advantage over ASTRID.

Comparison on the 10-taxon datasets. The 10-taxon datasets evolved under two different ILS levels-high and very high, and we explored performance on both true and estimated gene trees; see Figure 9. In general, ASTRID and ASTRAL-2 had very close accuracy on these data, but there were some cases where they had different accuracy levels. For example, on the high ILS
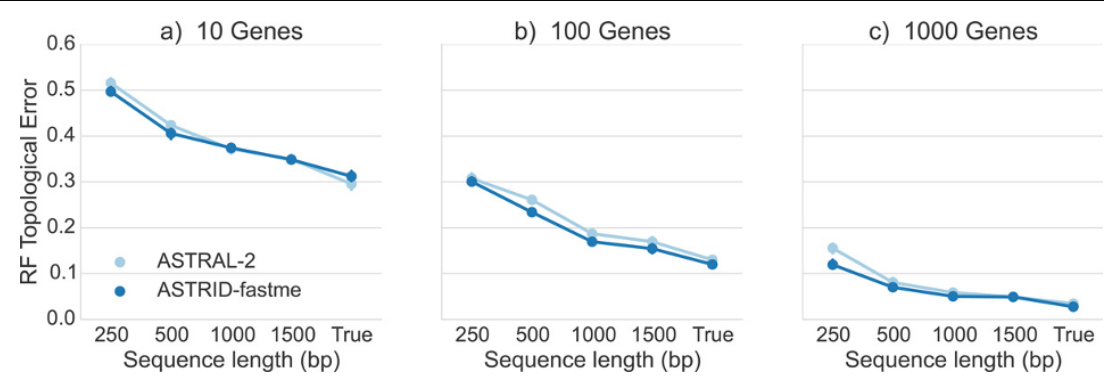

Figure 5 Performance on the avian simulated data with $1 \mathrm{X}$ species tree branch lengths, varying gene sequence length and number of genes. We report RF rates over 20 replicates.
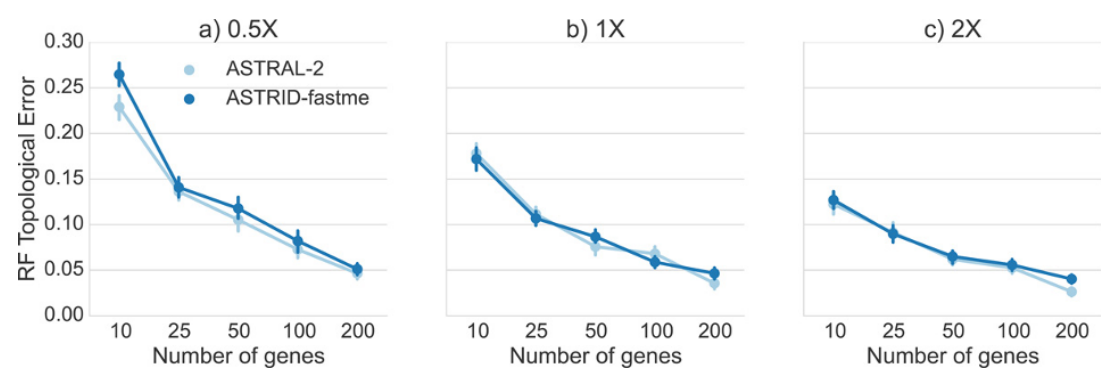

Figure 6 Comparison of methods on mammalian simulated datasets, varying ILS level and number of genes. We show average RF error rates and standard error bars for 20 replicates. Gene sequence alignments had 500 sites. Model conditions varied in ILS level from high (0.5X branch lengths) to low (2X branch lengths). 

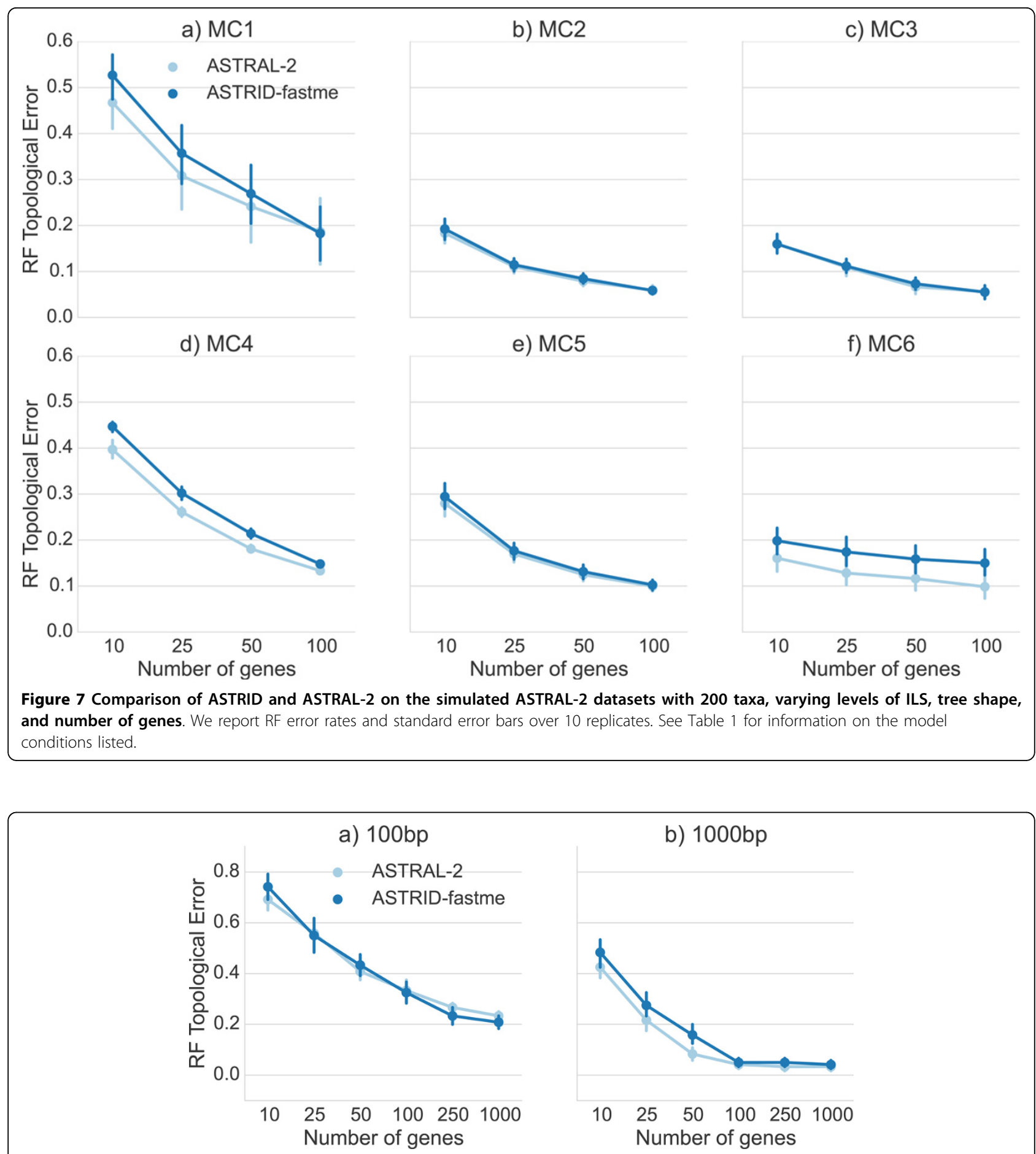

Figure 8 A comparison of ASTRID and ASTRAL-2 on the 15-taxon simulated datasets for two different sequence lengths. The 15-taxon datasets evolve down gene trees generated by a caterpillar tree with very high ILS (AD =82\%), the highest ILS condition explored in this study. We report mean RF rates and standard error over 10 replicates.

condition with estimated gene trees, ASTRAL-2 was more accurate than ASTRID for 200 genes, and ASTRID was more accurate than ASTRAL-2 on 25 genes.

\section{Performance on incomplete gene trees}

We explored the impact of missing data on ASTRAL-2 and ASTRID by deleting taxa from gene trees in the 50taxon datasets (MC8) from the ASTRAL-2 collection, 


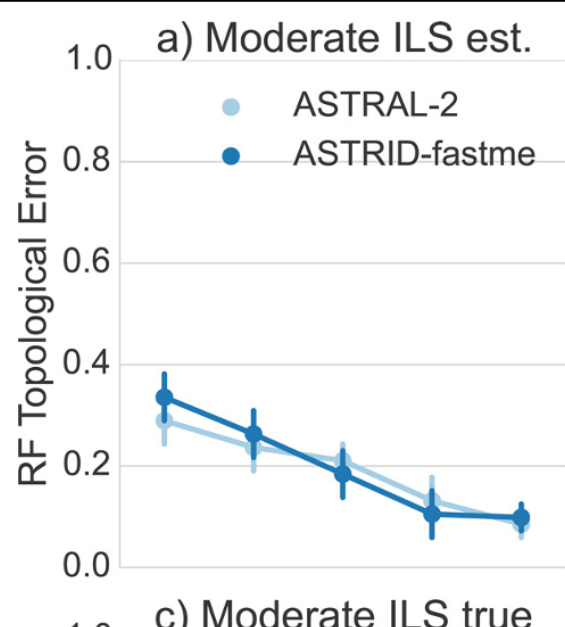

b) Very High ILS est.
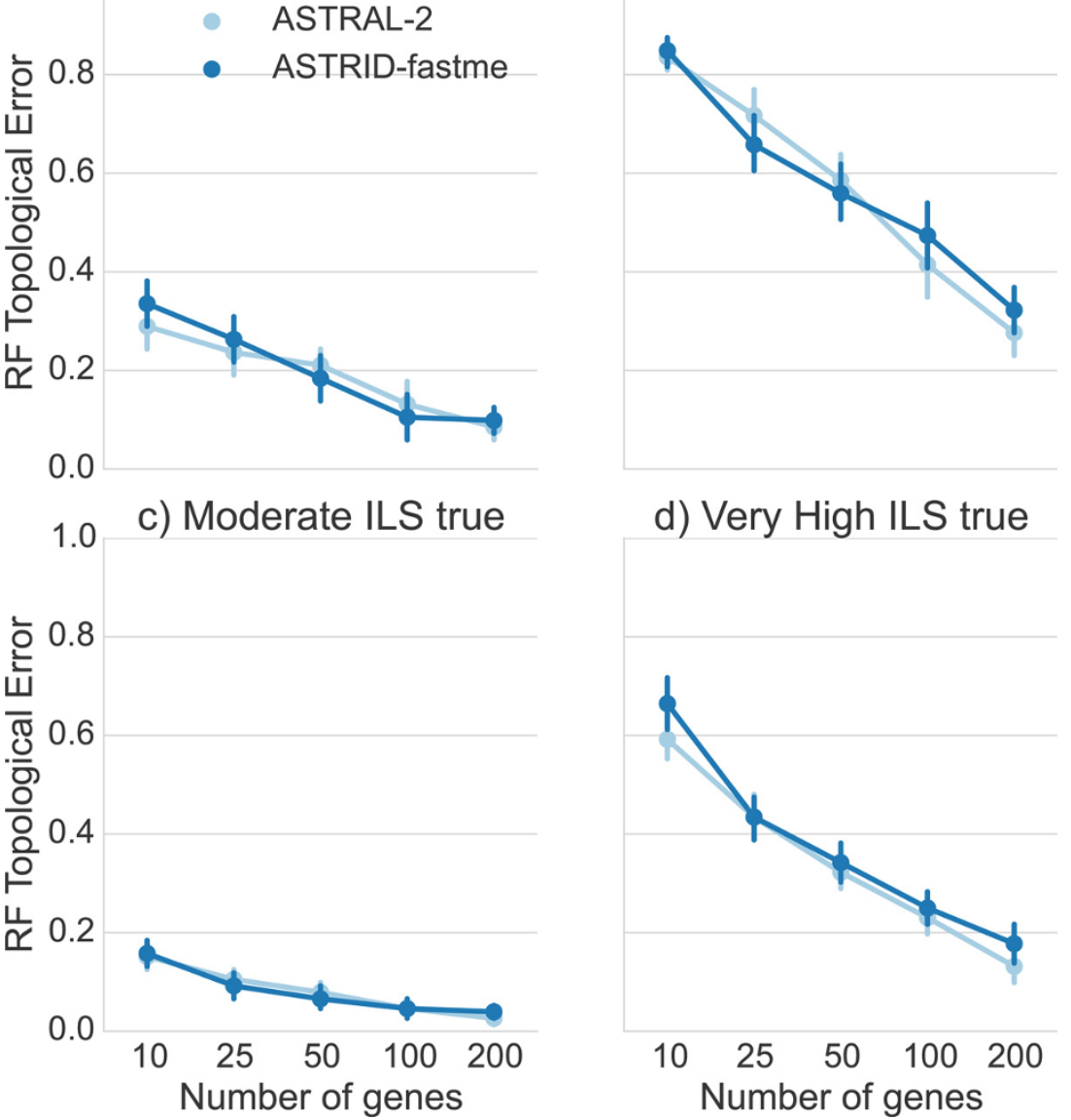

d) Very High ILS true

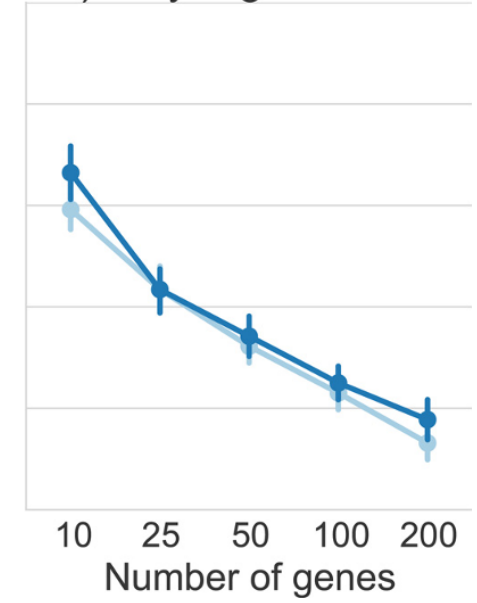

Figure 9 Results on true and estimated gene trees on 10-taxon datasets with two ILS levels (high and very high). All gene sequence alignments have $100 \mathrm{bp}$. We report RF rates and standard error bars over 20 replicates.

using $150 \mathrm{bp}$ per gene, and varying the number of genes and the amount of missing taxa; see Figure 10. ASTRAL-2 and ASTRID had very similar topological accuracy throughout these experiments. With low amounts of missing data (20\% to $40 \%$ missing taxa from each gene tree), both methods had very good accuracy (below $5 \%$ tree error) by 500 genes. With $60 \%$ of the taxa missing from each gene tree, the error rates increased for low numbers of genes (above 20\% RF error for up to 100 genes), but then declined to about $10 \%$ by 1000 genes. With $80 \%$ of the taxa missing from each gene (so that all gene trees have only 10 taxa out of 50), error rates were very high with 25 genes (at least $85 \% \mathrm{RF}$ ), but decreased quickly with increases in the number of genes, so that at 500 genes the error rate was $24 \%$, and then at most $18 \%$ at 1000 genes. The trends suggest that the error rates had not plateaued, and that adding additional incomplete gene trees should result in continued improvement.

\section{Analysis of the mammalian biological dataset}

We analyzed the mammalian biological dataset originally studied in [35]. The original dataset had 37 species and 447 genes, but there were 23 erroneous genes (as noted by [20]) which we removed before doing the analysis.

We obtained maximum likelihood gene trees and bootstrap replicates of these gene trees from [22]. We then analyzed these data using ASTRAL-2 and ASTRID +FastME and compared these analyses to previously published trees obtained using ASTRAL and MP-EST [7]. We then annotated the branches of the ASTRID +FastME and ASTRAL-2 trees with bootstrap support from 100 multi-locus bootstrapping (MLBS). The ASTRID+FastME and ASTRAL-2 trees were topologically identical to the ASTRAL tree and differed only in the bootstrap support; see Figure 11 for the ASTRID + FastME tree. On the other hand, the support for the placement of Scandentia-one of the major open questions about mammalian evolution-was very low, only 


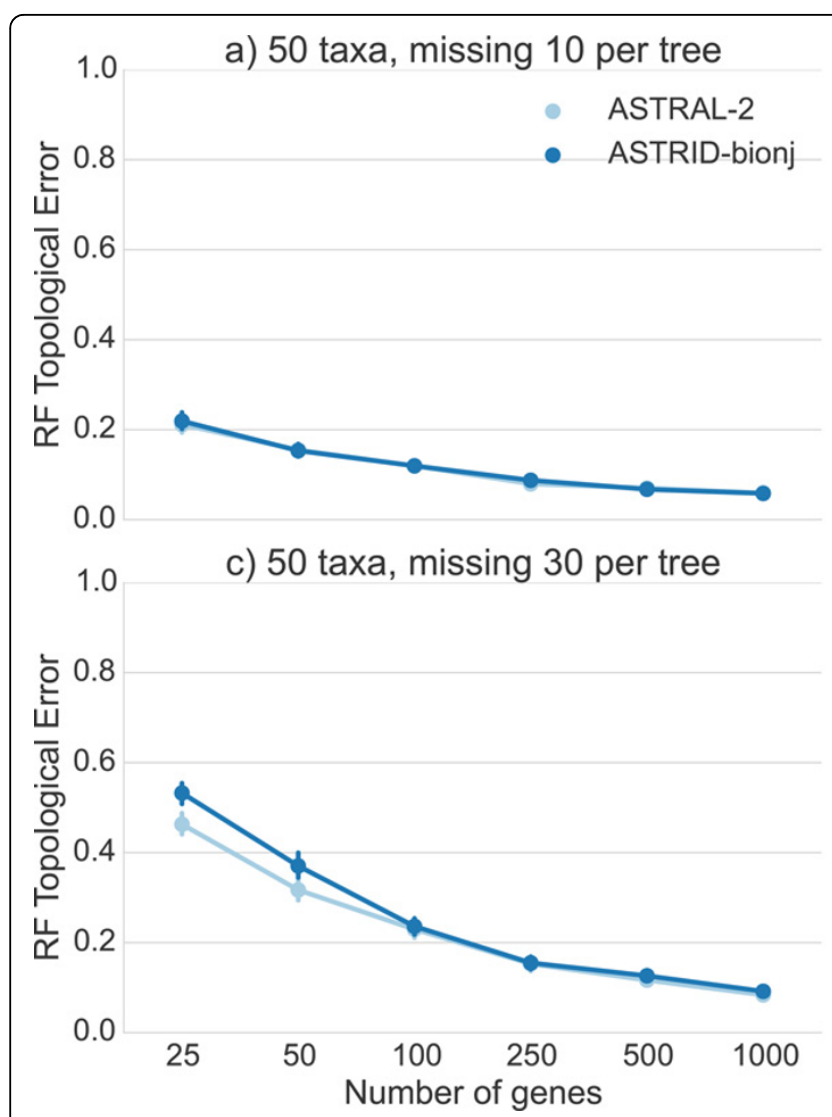

b) 50 taxa, missing 20 per tree

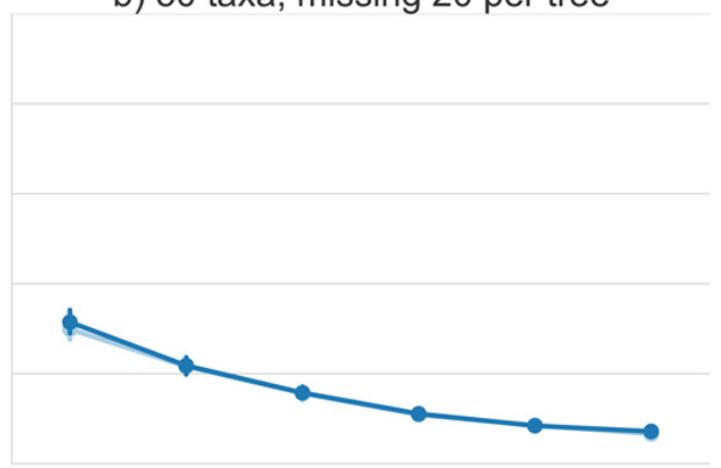

d) 50 taxa, missing 40 per tree

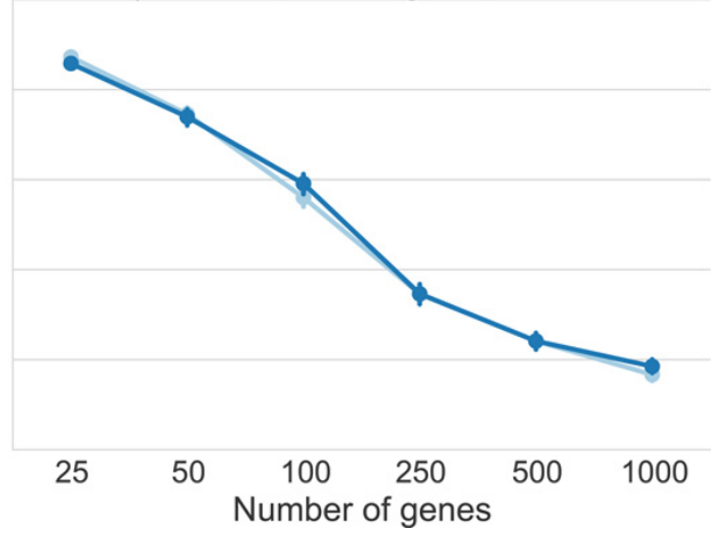

Figure 10 Results on 50-taxon ASTRAL-2 dataset (MC8) with missing taxa and sequence lengths of $\mathbf{1 5 0}$ bp. We report RF rates and standard error over 50 replicates.

47\% (ASTRAL-2 gave it 82\%). Hence, neither the ASTRID tree nor the ASTRAL-2 tree resolved the placement of Scandentia with high support.

\section{Running time results}

\section{Asymptotic running time}

ASTRID has two steps: the first step computes the distance matrix, and the second step uses a selected distancebased method to construct a tree from the distance matrix. When the input has $n$ species and $k$ genes, then calculating the distance matrix can be performed in $O\left(\mathrm{kn}^{2}\right)$ time. Distance-based tree estimation methods typically run in $O$ $\left(n^{2}\right)$ to $O\left(n^{3}\right)$ time, but this step no longer depends on $k$. Hence, the overall running time depends on the selected distance-based method, but is generally dominated by the first phase, especially for typical inputs, for which $k>>n$. Thus, under the assumption that $k>n$ and that ASTRID uses a distance-based method that runs in $O\left(n^{3}\right)$ time, ASTRID's running time is $O\left(\mathrm{kn}^{2}\right)$.

ASTRAL-2's scaling is more complicated to discuss. Asymptotically, ASTRAL-2 runs in $O\left(n k|X|^{2}\right)$ time, where $n$ is the number of species, $k$ is the number of genes, and $X$ is a set of bipartitions it computes to constrain the search space. The size of $X$ is not bounded by a polynomial in the input size, and the technique that ASTRAL-2 uses means that $X$ can be large under conditions with high ILS. Thus the asymptotic running times of ASTRAL-2 and ASTRID (used with various distance methods) are quite different.

\section{Running times on simulated data}

In practice, creating the distance matrix took the majority of the running time. On 1000 taxa, creating the distance matrix took several minutes to several hours, depending on the number of genes, but running FASTME took less than one second regardless of the number of genes. However, PhyD* methods were much slower than FASTME; on 1000 taxa, running any of the PhyD* methods took approximately 40 minutes (data not shown). ASTRID depends on FastME, PhyD*, and Dendropy [36].

We recorded running times for ASTRAL-2, ASTRIDFastME, and NJst, on avian simulated datasets with high ILS (1X), as we varied the number of genes (see Figure 12). Note that ASTRID-FastME was by far the fastest of the three methods, and NJst was the slowest. However, the trends suggest that NJst will be faster than ASTRAL-2 for larger numbers of genes. Note also that ASTRID-FastME 


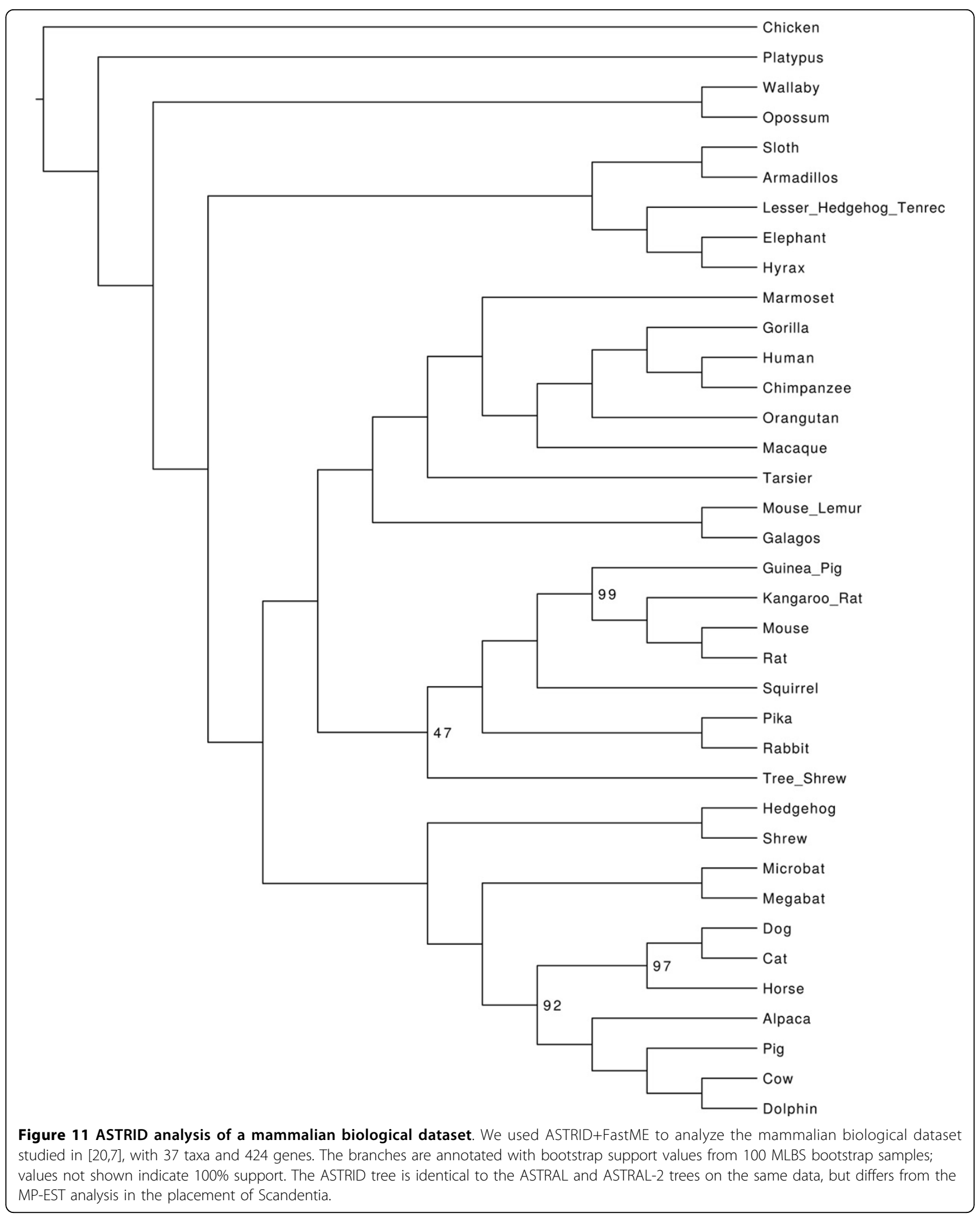




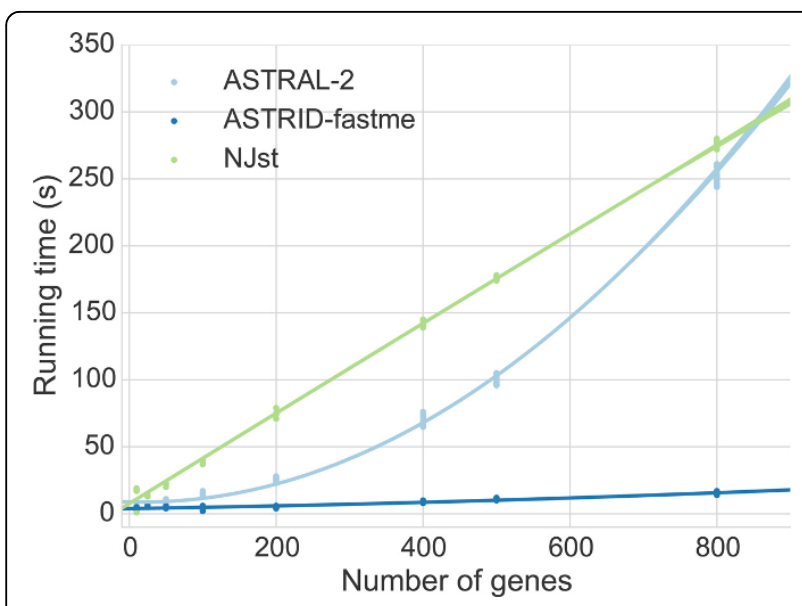

Figure 12 Scatterplot of running times for ASTRID-FastME, ASTRAL-2, and NJst, on avian high ILS (1X) simulated datasets, varying number of genes. We show running time for 20 replicates of each number of genes. The quadratic dependence of ASTRAL-2's running time is clearly contrasted with the linear dependence of both ASTRID and NJst. Experiments were run on a single core of a 2.7 GHz Intel Xeon processor.

and NJst both scaled linearly with the number of genes, but that ASTRAL-2's running time scaled superlinearly.

We recorded running times for two variants of ASTRID (one using FastME and the other using BioN)*), and compared them to ASTRAL-2 on ASTRAL-2 simulated datasets with 1000 taxa (MC11) as we varied the number of genes (Figure 13) and for 500-gene datasets in which we varied the number of taxa (MC 2 and 7-10, see Figure 14). The relative running times show that all methods were very fast for smaller datasets, but were clearly distinguished on the larger datasets, where ASTRID-FastME was much faster than ASTRID-BioNJ" and both variants of ASTRID were much faster than ASTRAL-2. For example, on the dataset with 1000 genes and 1000 taxa, ASTRID-FastME finished in 33 minutes, ASTRID-BioNJ finished in 1 hour and 10 minutes, and ASTRAL-2 finished in 12 hours and 30 minutes.

\section{Running times on biological data}

We recorded running times for ASTRID-FastME and ASTRAL-2 on the mammalian biological dataset. Both methods took 6 seconds for a single bootstrap replicate on one core of a $2.7 \mathrm{GHz}$ Intel Xeon processor with 424 genes and 37 taxa.

\section{Discussion}

A few trends are apparent upon examining the data as a whole. ASTRAL-2 and ASTRID had, for the most part, very similar levels of accuracy, while MP-EST was consistently less accurate. However, there were cases where ASTRID and ASTRAL-2 have small but detectably different levels of accuracy. One intriguing trend in the data is the improvement of ASTRAL-2 over ASTRID on high ILS datasets; see Figures 6, 7, 8, and 9. In particular, Figures 6 and 7 suggest that increases in ILS should favor ASTRAL-2 over ASTRID. Yet, ASTRID is consistently at least as accurate as ASTRAL-2 on the avian datasets, which have moderate to very high levels of ILS (Fig. 4). Thus, ILS level might have an impact on the relative accuracy of the two methods, but it is not a determining favor. Similarly, neither method dominates the other based on the number of taxa, number of genes, or amount of gene tree estimation error. Thus, it is very difficult to characterize the conditions under which each method is likely to have an advantage over the other. However, even for the cases where there are differences in accuracy, in general the differences are fairly small. Thus, the main difference between the two methods is computational efficiency, where ASTRID is clearly faster. ASTRID has the biggest running time advantage over ASTRAL-2 for large numbers of gene trees, since ASTRID scales linearly in the number of genes while ASTRAL scales superlinearly. This makes
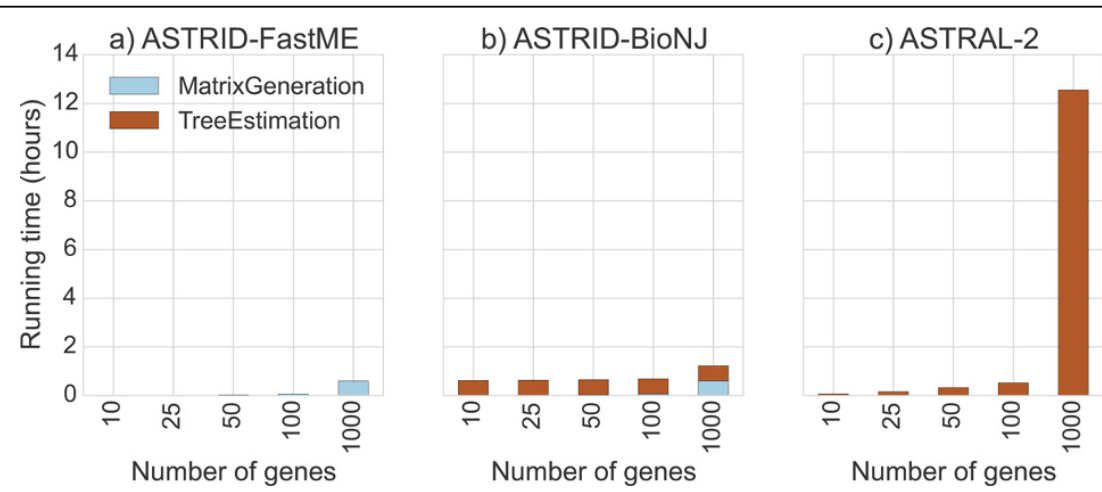

Figure 13 Running time on the ASTRAL-2 simulated datasets with 1000 taxa (MC11), varying number of genes. We show results for the each of the ASTRID steps - matrix generation and tree estimation. We compare ASTRID used with two ways of computing the trees: FastME and BioNJ*. Experiments were run on a single core of a $2.7 \mathrm{GHz}$ Intel Xeon processor. 

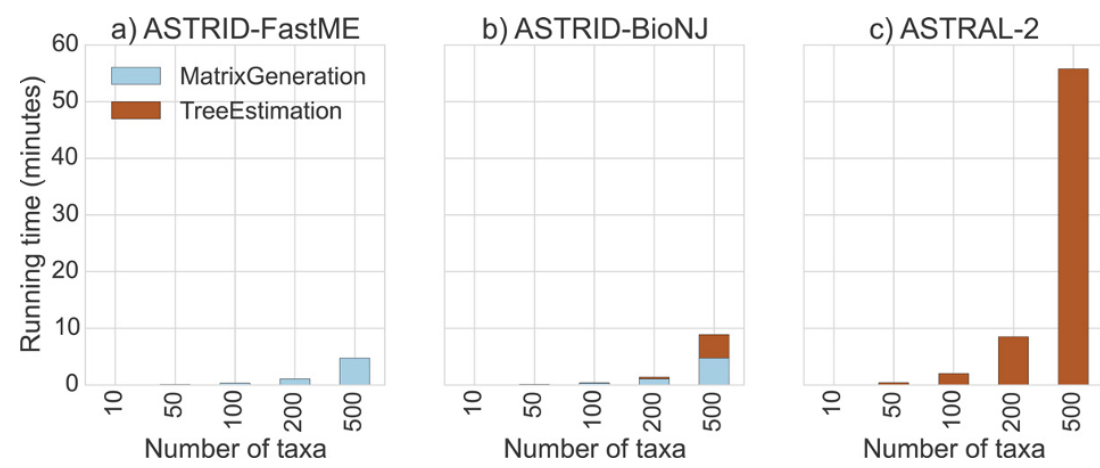

Figure 14 Running time of two ASTRID methods on the ASTRAL-2 simulated dataset with 500 genes, varying number of taxa. We show results on a single replicate of model conditions MC2 and 7-10 from the ASTRAL-2 collection. Experiments were run on a single core of a $2.7 \mathrm{GHz}$ Intel Xeon processor.

ASTRID an especially good method for genome-scale datasets that have a large number of genes.

\section{Conclusion}

ASTRID is a fast and highly accurate method for species tree estimation that is robust to high levels of ILS, and provably statistically consistent under the multi-species coalescent model. Like ASTRAL-2, ASTRID can analyze datasets with unrooted gene trees, an advantage that the two methods have over many other methods (e.g., MPEST) that can only be run on rooted gene trees. ASTRID (like NJst) runs in time that is polynomial in the number of gene trees and species, but ASTRAL-2 and other leading coalescent-based methods do not have this guarantee. Thus, ASTRID has many desirable theoretical properties compared to existing methods.

From an empirical viewpoint, ASTRID is also extremely fast and can analyze very large datasets in minutes, where other methods either cannot run or take hours. In particular, ASTRID is much faster than ASTRAL-2, especially on datasets with many genes and large numbers of species. ASTRID also produces more accurate trees than MP-EST and NJst, and is competitive with ASTRAL-2 in terms of accuracy.

However, even better (more accurate) results might be obtained through more extensive modifications to the ASTRID algorithm design. In particular, the accuracy of the tree depends on the particular distance-based method that is used. New distance-based phylogeny estimation methods, such as the absolute fast converging methods [37-40], might provide improved accuracy for very large datasets. Another important direction is developing additional methods for estimating species trees from distance matrices that have good accuracy when the distance matrix has missing data. As we saw here, FastME produced more accurate trees than the PhyD" methods, but it could only be applied to distance matrices without any missing data. An extension of
FastME to enable it to handle incomplete distance matrices would also be of great interest.

This study can be expanded in several directions. Future work should more carefully investigate the conditions under which ASTRID is more reliable than ASTRAL-2, and explore performance on more biological datasets. This study also only investigated relatively long sequences; a subsequent study should investigate the relative and absolute accuracy of ASTRID and other methods on very short sequences, since recombination-free loci can be very short [32]. In addition, this study only examined datasets with a single individual per species, yet ASTRID (like NJst) can be run on datasets with multiple individuals; future work should evaluate the absolute and relative accuracy of ASTRID and other methods on such data. This study showed that ASTRID performed well in terms of species tree topology estimation, but we did not explore its accuracy with respect to the estimation of coalescent branch lengths; future work will need to explore how well ASTRID estimates these numeric parameters. Finally, it may well be that ASTRID will be most useful as a starting tree for use within more computationally intensive analyses, including Bayesian MCMC analyses (e.g., "BEAST) or maximum likelihood analyses.

\section{Availability of supporting data}

All datasets used in this study are available from prior publications. ASTRID is available in open source form on github at http://pranjalv123.github.io/ASTRID. Supporting materials are available online at http://pranj.al/ASTRID.

\footnotetext{
Abbreviations

AD: Average distance

ILS: Incomplete lineage sorting

MSC: Multi-species coalescent

RF: Robinson-Foulds
}

Competing interests

The authors declare that they have no competing interests. 


\section{Authors' contributions}

PV implemented ASTRID, performed experiments, wrote the first draft, and analyzed the data. TW designed the study, analyzed the data, and wrote the final draft.

\section{Acknowledgements}

PV was supported by the Roy J. Carver graduate fellowship from the University of Illinois at Urbana-Champaign, College of Engineering. TW was supported by National Science Foundation grant DBI-1461364. PV and TW were both supported by the College of Engineering at the University of Illinois at Urbana-Champaign through a gift from the Grainger Foundation. Publication costs for this article were funded by the corresponding author's institution.

This article has been published as part of BMC Genomics Volume 16 Supplement 10, 2015: Proceedings of the 13th Annual Research in Computational Molecular Biology (RECOMB) Satellite Workshop on Comparative Genomics: Genomics. The full contents of the supplement are available online at http://www.biomedcentral.com/bmcgenomics/ supplements/16/S10

Published: 2 October 2015

\section{References}

1. Maddison W: Gene trees in species trees. Syst Biol 1997, 46(3):523-536.

2. Kingman JFC: On the genealogy of large populations. J Appl Prob 1982, 19:27, doi:10.2307/3213548

3. Roch S, Steel M: Likelihood-based tree reconstruction on a concatenation of alignments can be statistically inconsistent. Theoretical Population Biology 2015, 100:56-62.

4. Degnan JH, Rosenberg NA: Gene tree discordance, phylogenetic inference and the multispecies coalescent. Trends Ecol Evol 2009, 24(6):332-340, doi:10.1016/j.tree.2009.01.009.

5. Liu L, Yu L, Kubatko L, Pearl DK, Edwards SV: Coalescent methods for estimating phylogenetic trees. Mol Phylogenet Evol 2009, 53(1):320-328.

6. Knowles LL, Kubatko L: Estimating Species Trees: Practical and Theoretical Aspects Wiley-Blackwell, Hoboken, NJ; 2011.

7. Mirarab S, Reaz R, Bayzid MS, Zimmermann T, Swenson MS, Warnow T: ASTRAL: genome-scale coalescent-based species tree estimation. Bioinformatics 2014, 30:541-548, doi:10.1093/bioinformatics/btu462.

8. Mirarab S, Warnow T: ASTRAL-II: coalescent-based species tree estimation with many hundreds of taxa and thousands of genes. Bioinformatics 2015, 31(12):44-52[http://bioinformatics.oxfordjournals.org/ content/31/12/i44.full.pdf+html], doi:10.1093/bioinformatics/btv234..

9. Heled J, Drummond AJ: Bayesian inference of species trees from multilocus data. Mol Biol Evol 2010, 27:570-580, doi:10.1093/molbev/ msp274.

10. Liu L, Pearl DK: Species trees from gene trees: reconstructing Bayesian posterior distributions of a species phylogeny using estimated gene tree distributions. Syst Biol 2007, 56:504-514, doi:10.1080/10635150701429982.

11. Larget BR, Kotha SK, Dewey CN, Ané C: BUCKy: Gene tree/species tree reconciliation with Bayesian concordance analysis. Bioinformatics 2010, 26:2910-2911, doi:10.1093/bioinformatics/btq539.0912.4472.

12. Dasarathy G, Nowak R, Roch S: Data requirement for phylogenetic inference from multiple loci: A new distance method. IEEE/ACM Trans Comp Biol Bioinformatics 2015, 12:422-432, DOI: 10.1109/TCBB.2014.2361685.

13. Liu L, Yu L, Edwards SV: A maximum pseudo-likelihood approach for estimating species trees under the coalescent model. BMC Evol Biol 2010, 10:302, doi:10.1186/1471-2148-10-302.

14. Liu L, Yu L: Estimating species trees from unrooted gene trees. Syst Biol 2011, 60(5):661-667, doi:10.1093/sysbio/syr027.

15. Bryant $D$, Bouckaert R, Felsenstein J, Rosenberg NA, RoyChoudhury A: Inferring species trees directly from biallelic genetic markers: bypassing gene trees in a full coalescent analysis. Mol Biol Evol 2012, 29(8):1917-1932.

16. Liu L, Yu L, Pearl DK, Edwards SV: Estimating species phylogenies using coalescence times among sequences. Syst Biol 2009, 58(5):468-477, doi:10.1093/sysbio/syp031.

17. Kubatko L, Carstens BC, Knowles LL: STEM: Species tree estimation using maximum likelihood for gene trees under coalescence. Bioinformatics 2009, 25:971-973.
18. Chifman J, Kubatko L: Quartet inference from SNP data under the coalescent model. Bioinformatics 2014 [http://bioinformatics.oxfordjournals. org/content/early/2014/08/27/bioinformatics.btu530.full.pdf+html], doi:10.1093/bioinformatics/btu530.

19. Saitou N, Nei M: The neighbor-joining method: a new method for reconstructing phylogenetic trees. Mol Biol Evol 1987, 4:406-425, doi: citeulike-article-id:93683.

20. Mirarab S, Bayzid MS, Boussau B, Warnow T: Statistical binning enables an accurate coalescent-based estimation of the avian tree. Science 2014, 346(6215):1250463.

21. Robinson DF, Foulds LR: Comparison of phylogenetic trees. Math Biosci 1981, 53:131-147

22. Mirarab S, Bayzid MS, Bossau B, Warnow T: Statistical binning improves species tree estimation in the presence of gene tree heterogeneity. Science 2014, 346(6215):1250463.

23. Bayzid MS, Mirarab S, Warnow T: Weighted statistical binning: enabling statistically consistent genome-scale phylogenetic analyses. PLOS One 2014.

24. Jarvis ED, Mirarab S, Aberer AJ, Li B, Houde P, Li C, Ho SY, Faircloth BC, Nabholz B, Howard JT: Whole-genome analyses resolve early branches in the tree of life of modern birds. Science 2014, 346(6215):1320-1331.

25. Song S, Liu L, Edwards SV, Wu S: Resolving conflict in eutherian mammal phylogeny using phylogenomics and the multispecies coalescent model. Proc Natl Acad Sci USA 2012, 109(37):14942-14947.

26. Mallo D, Oliviera Martins L, Posada D: SimPhy: Comprehensive Simulation of Gene, Locus and Species Trees at the Genome-wide Level [https://github.com/ adamallo/SimPhy].

27. Price MN, Dehal PS, Arkin AP: FastTree 2-Approximately maximumlikelihood trees for large alignments. PLOS ONE 2010, 5(3), doi:10.1371/ journal.pone.0009490.

28. Criscuolo A, Gascuel O: Fast NJ-like algorithms to deal with incomplete distance matrices. BMC Bioinformatics 2008, 9:166, doi:10.1186/1471-2105-9-166.

29. Desper R, Gascuel O: Fast and accurate phylogeny minimum-evolution principle. J Comput Biol 2002, 9:687-705, doi:10.1089/106652702761034136.

30. Bayzid MS, Hunt T, Warnow T: Disk covering methods improve phylogenomic analyses. BMC Genomics 2014, 15(Suppl 6):7.

31. Mirarab S, Bayzid MS, Warnow T: Evaluating summary methods for multilocus species tree estimation in the presence of incomplete lineage sorting. Syst Biol 2014, 63.

32. Gatesy JP, Springer MS: Phylogenetic analysis at deep timescales: Unreliable gene trees, bypassed hidden support, and the coalescence/ concatalescence conundrum. Mol Phylog Evol 2014, 80:231-266.

33. Bayzid MS, Warnow T: Naive binning improves phylogenomic analyses. Bioinformatics 2013, 29(18):2277-84, doi:10.1093/bioinformatics/btt394.

34. Roch $S$, Warnow T: On the robustness to gene tree estimation error (or lack thereof) of coalescent-based species tree methods. Syst Biol 2015, 64(4):663-676.

35. Song S, Liu L, Edwards SV, Wu S: Resolving conflict in eutherian mammal phylogeny using phylogenomics and the multispecies coalescent model. Proc Natl Acad Sci USA 2012, 109(37):14942-14947.

36. Sukumaran J, Holder MT: DendroPy: A Python library for phylogenetic computing. Bioinformatics 2010, 26(12):1569-1571.

37. Roch S: Towards extracting all phylogenetic information from matrices of evolutionary distances. Science 2010, 327(5971):1376-1379.

38. Warnow T, Moret BME, St John K: Absolute phylogeny: true trees from short sequences. Proc 12th Ann ACM/SIAM Symp Discrete Algs (SODA01) SIAM Press, Philadelphia, PA; 2001, 186-195.

39. Gronau I, Moran S, Snir S: Fast and reliable reconstruction of phylogenetic trees with indistinguishable edges. J Random Struct Algs 2012, 40(3):350-384, doi $=10.1002 /$ rsa.20372.

40. Nakhleh L, Roshan U, St John K, Sun J, Warnow T: Designing fast converging phylogenetic methods. Bioinformatics 2001, 17(suppl 1):190-198, doi:10.1093/ bioinformatics/17.suppl 1.S190

doi:10.1186/1471-2164-16-S10-S3

Cite this article as: Vachaspati and Warnow: ASTRID: Accurate Species TRees from Internode Distances. BMC Genomics 2015 16(Suppl 10):S3. 